\title{
LMS filter based frequency domain system identification of mass - spring - damper system with varying dynamics
}

\author{
Winston Netto \\ Manipal Institute of Technology, \\ Manipal Academy of Higher Education, \\ Manipal, Karnataka, India
}

\author{
S. Meenatchi Sundaram \\ Manipal Institute of Technology, \\ Manipal Academy of Higher Education, \\ Manipal, Karnataka, India
}

\author{
Eloy Pena Asensio \\ Department of Aerospace Engineering \\ Technical University of Madrid, \\ Madrid, Spain \\ C.R. Srinivasan \\ Manipal Institute of Technology, \\ Manipal Academy of Higher Education, \\ Manipal, Karnataka, India
}

Received: March 2, 2021. Revised: November 14, 2021. Accepted: December 22, 2021. Published: January 17, 2022.

\begin{abstract}
All practical and real-time systems are non-linear and dynamic in nature. In the majority of studies associated with systems, it is assumed that the system is linear and the dynamics of the system is remaining constant. Though these assumptions help in easier mathematical formulations of the systems, it also imposes a lot of restrictions on understanding the system completely and its behavior in depth. In this study, the focus is on capturing the varying dynamics of Mass Spring - Damper system using a frequency domainbased system identification approach. The system identification technique is based on an adaptive filter which is implemented through MATLAB software.
\end{abstract}

Keywords-System Identification, Frequency domain, Adaptive filter, Varying Dynamics, Least Mean - Square (LMS) algorithm, TRMS

\section{INTRODUCTION}

$\mathrm{T}$ The control theory when applied to a dynamic system can become more challenging if the dynamics associated with plant is varying with time. For proper control of the system, the changes in dynamics need to be captured online. The online system identification techniques can be used for capturing the dynamics of the system in real - time. Accordingly, this work focuses on exploring the possibility of online identification for capturing the changes in dynamics of the system in real - time. The system considered is a simple mass - spring - damper mechanical system.

The identification routines can be broadly classified into two main divisions: nonparametric estimation methods and parametric estimation methods [1]. Parametric methods need knowledge about the plant model in prior, whereas nonparametric methods do not require any prior knowledge as they are based on input-output frequency response of the system under study. The major advantage of using frequency domain based technique for online identification are - better rejection of noise, reduced data size and good model validation. The model is obtained from the input and output signals of the plant, generated by a known excitation signal. For the identification of a system, the selection of excitation signal is very critical. Most widely used excitation signals are filtered Gaussian white noise, random binary noise, and pseudorandom binary sequence (PRBS). Also periodic excitation signals such as multisines and swept sinusoids are also used.

In literature, the techniques used for frequency response analysis includes, fast Fourier transform (FFT), discrete Fourier transform (DFT), wavelet transformations and adaptive filters [2]-[3]. The disadvantages of using FFT approach for frequency response analysis is that the system dynamics should not change during the measurement of response [1]. Other drawbacks of such techniques are the array and buffer size allocation for calculations, noise rejection capabilities and requirements for post processing of data. The above limitations can be addressed using adaptive filters. Adaptive filters use computationally less intensive least mean square algorithm which makes it favorable for real - time applications.

Considering the various pros and cons of various techniques available for online frequency domain identification of systems, a less complex nonparametric adaptive filter based method is used in this work for capturing the varying dynamics of a benchmark mass - 
spring - damper system. The identification method and implementation procedure used for this system can be used to capturing the dynamics of other time varying systems.

The paper is organized as follows: the method of system identification using adaptive filter is explained in section II. A very brief description about the plant is given in section III. The procedure for implementing system identification for the mass - spring - damper system is explained in section IV. And, the results obtained and future scope of the work are discussed in section $\mathrm{V}$.

\section{ADAPTIVE FILTER BASED ONLINE SYSTEM IDENTIFICATION}

The parameters and states of the system in operation can be estimated using online estimation techniques based on the new available data. The basic structure of system identification using adaptive filter is shown in Fig. 1.

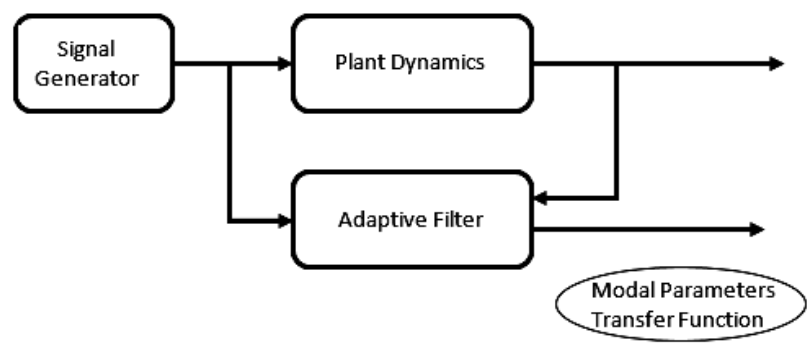

Fig. 1 Adaptive filter based system identification

The signal generator is used to generate an appropriate excitation signal which can perturb the system to extract the input - output data required for identification. Generator can be a hardware device or a software based generator which is connected to plant through an interface card. Plant dynamics block represents the unknown plant model for which identification has to be performed and adaptive filter is the technique to identify the model from the input - output data.

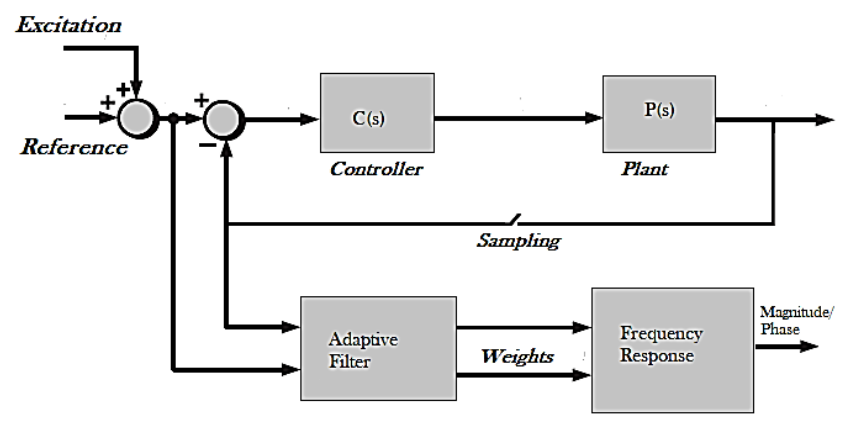

Fig. 2 Frequency response analysis using adaptive filter

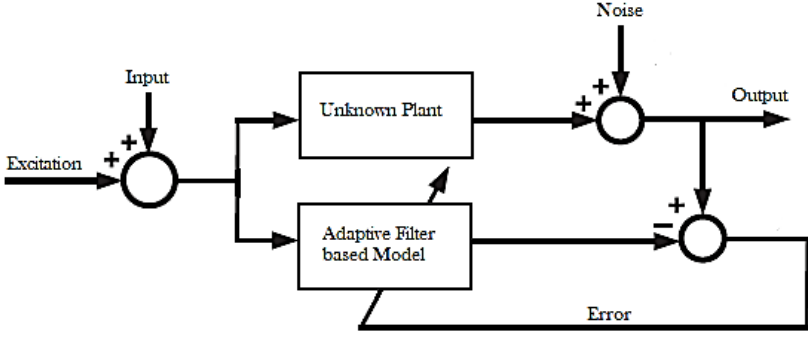

Fig. 3 Online system identification using adaptive filter

A block diagram describing frequency response analysis using adaptive filter is shown in Fig 2 [1] and Fig 3 shows the implementable scheme of online system identification using adaptive filter method. As shown, the input to the unknown plant and the adaptive model is periodically excited through a proper excitation signal which results in an output signal from unknown plant and adaptive filter. A noise signal is added to the output of the plant and that results in the desired output. The plant output and adaptive filter output are compared and the error is used to update the weights so that both model converges and zero error is produced.

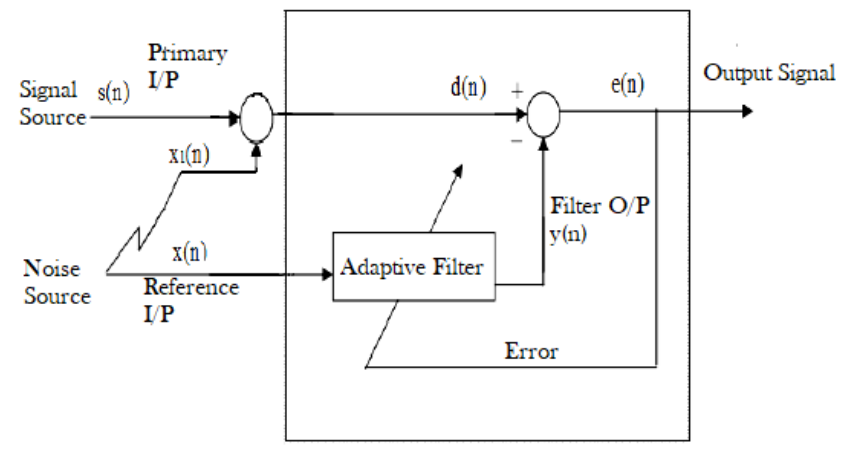

Fig. 4 Adaptive filter noise cancellation system

Adaptive filter is a band stop filter and the bandwidth can be adjusted around the center frequency, this can track the input signal. The structure is as shown in Fig. 4 [3]. The estimated signal or reconstructed $y(n)$ is compared with the signal $d(n)$. Any error $e(n)$ in estimation of the input signal is given as input to the accumulator that uses LMS algorithm. Through continuous adaptation of the weights the error $\mathrm{e}(\mathrm{n})$ is driven to zero. The rate of convergence and also stability of the filter is determined by the adaptation gain $\mu$. Simple to implement, low computational complexity, and possibility of real-time operation are the advantages of LMS algorithm.

The estimated signal y (n) can be defined as [3]:

$$
y(n)=\vec{u}^{T}(n) \vec{w}(n)
$$

where $\vec{u}(n)$ is the filter input vector given by,

$$
\vec{u}(n)=[x(n) x(n-1) \ldots x(n-N+1)]^{T}
$$


and $\vec{w}(n)$ is the filter coefficients vector given by,

$$
\vec{w}(n)=\left[w_{0}(n) w_{1}(n) \ldots w_{N-1}(n)\right]^{T}
$$

The error signal $\mathrm{e}(\mathrm{n})$ is calculated by,

$$
e(n)=d(n)-y(n)
$$

The filter coefficients are updated as follows,

$$
\vec{w}(n+1)=(1-\mu) \vec{w}(n)+\mu e(n) \vec{u}(n)
$$

\section{PLANT WITH VARYING DYNAMICS}

The system considered for studying the online identification of varying dynamic system is a bench mark underdamped mass - spring - damper (MSD) system. This makes it possible for the easy understanding of the system and the validation of identified model is much simpler. A typical MSD system is shown in Fig 4.

The equation governing mass-spring-damper system is a 2nd-order differential equation:

$$
F(t)=m \ddot{x}+b \dot{x}+k
$$

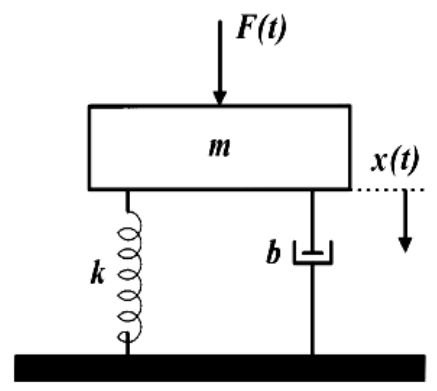

Fig. 5 Mass - Spring - Damper System

Assuming initial conditions to be zero and by taking the Laplace transform, the transfer function model is obtained as:

$$
G(s)=\frac{X(s)}{F(s)}=\frac{1}{m s^{2}+b s+k}
$$

Comparing equation (7) with a standard second order transfer function, the damping ratio and natural frequency of oscillation of the system can be expressed as follows:

$$
\begin{gathered}
\text { Damping ratio, } \zeta=\frac{b}{2 \sqrt{k m}} \\
\text { Natural frequency, } \omega_{n}=\sqrt{\frac{k}{m}}
\end{gathered}
$$

With the above system, two cases have been studied. MSD system with (i) fixed mass and (ii) variable mass. For case (i), following parameters were arbitrarily chosen for describing the system:

$$
\text { Mass, } \mathrm{m}=0.01 \mathrm{~kg}
$$

Spring constant, $\mathrm{k}=1$
Damping constant, $\mathrm{b}=0.04$

For case (ii), spring constant and damping constant was same as in case (i). The mass of the system is varied at regular intervals of time as follows:

Table I. Mass at different intervals of time

\begin{tabular}{|c|c|}
\hline Time Intervals (secs) & Mass $(\mathrm{kg})$ \\
\hline 0 to 20 & 0.02 \\
\hline 20.2 to 40 & 0.015 \\
\hline 40.2 to 60 & 0.01 \\
\hline 60.2 to 80 & 0.0085 \\
\hline
\end{tabular}

\section{SIMULATION AND RESULTS FOR LMS ALGORITHM BASED IDENTIFICATION}

An adaptive filter using least mean square (LMS) algorithm is used for the system identification. Various steps involved in this process is as follows, generation of proper excitation signal, exciting the system with designed signal and obtaining the of output response, using the input output data from plant as well as adaptive filter, the weights are updated and finally made to converge, and the identified model is validated by exciting it with a standard input. These various steps are described in the subsequent sections of this chapter.

\section{A. Generation of excitation signal}

In order to perform proper identification, the first step is to design an appropriate excitation signal. The excitation signal chosen is a swept sine signal which exhibits an instantaneous frequency that increases exponentially with time [3]. The signal is also known as exponential chirp signal. The signal is defined as:

$$
s=\operatorname{Asin}\left(2 \pi f_{0}\left(\frac{k^{t}-1}{\ln k}\right)+\Phi_{0}\right)
$$

where $A$ is the amplitude of wave, $f_{0}$ is the start frequency at $\mathrm{t}=0, \Phi_{0}$ is the initial phase at $\mathrm{t}=0$ and $k$ is the rate of exponential increase in frequency.

The parameter $k$ depends on stop frequency $\left(f_{T}\right)$ and the time length.

$$
k=\left(\frac{f_{T}}{f_{0}}\right)^{\frac{1}{T}}
$$

then instantaneous frequency $\left(f_{i}\right)$ is,

$$
f_{i}=f_{0} k^{t}
$$

and the instantaneous phase $\left(\Phi_{i}\right)$ is,

$$
\Phi_{i}=f_{0}\left(\frac{k^{t}-1}{\log (k)}\right)
$$



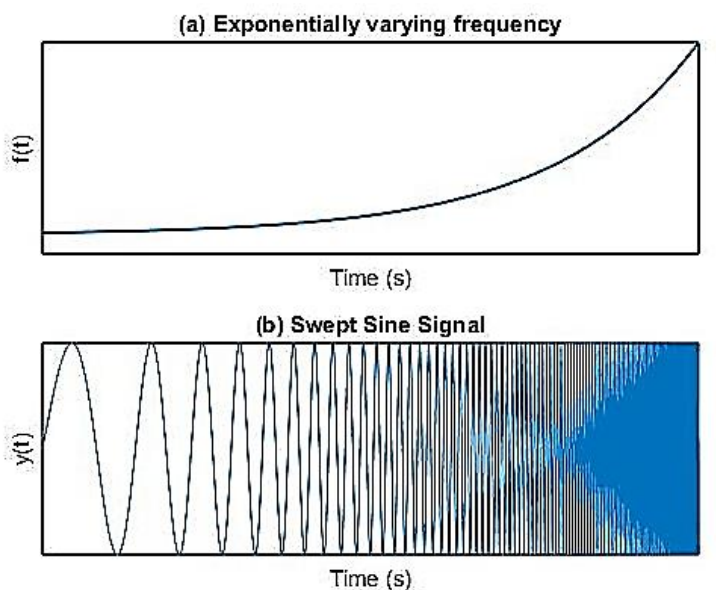

Fig. 6 (a) Exponential variation of frequency (b) Swept sine signal

The chirp signal used for excitation was generated using equation (8) and is as shown in Fig 6(b). The initial frequency is $f_{0}=1 \mathrm{~Hz}$ and final frequency is $f_{T}=100 \mathrm{~Hz}$. Time length chosen is 5 seconds.

\section{B. Identification procedure}

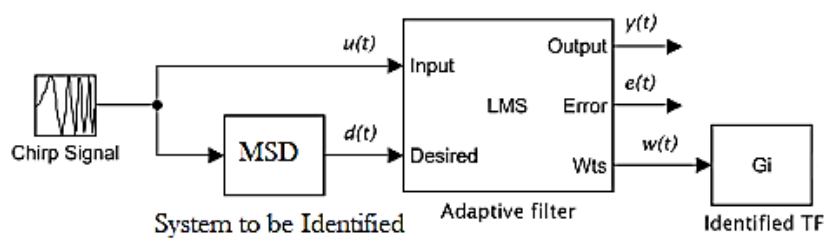

Fig. 7 Adaptive filter based identification

The chirp signal is given as input to both adaptive filter as well as to the system that need to be identified. From the desired output (output from plant) and output of filter, the error signal is generated and weights of filter are adjusted fixed dynamics, with fixed mass and case (ii) - varying dynamics, with mass of the system varying at regular intervals.

Case (i): The MSD system has been excited with the chirp signal and the adaptive filter has been applied generating a response signal $\mathrm{y}(\mathrm{t})$ and the estimated filter weights $\mathrm{w}$, which is a vector with eleven values. As shown in Fig. 8, the signal produced by the filter is quite similar to the desired signal. The error is more pronounced in the first moments but decreases rapidly with the advance of time. The estimated filter weights of the identified model are used to get the plant model.

The response of plant and filter for chirp signal excitation are as shown in Fig. 8. The model obtained using identification is validated using step response as shown in

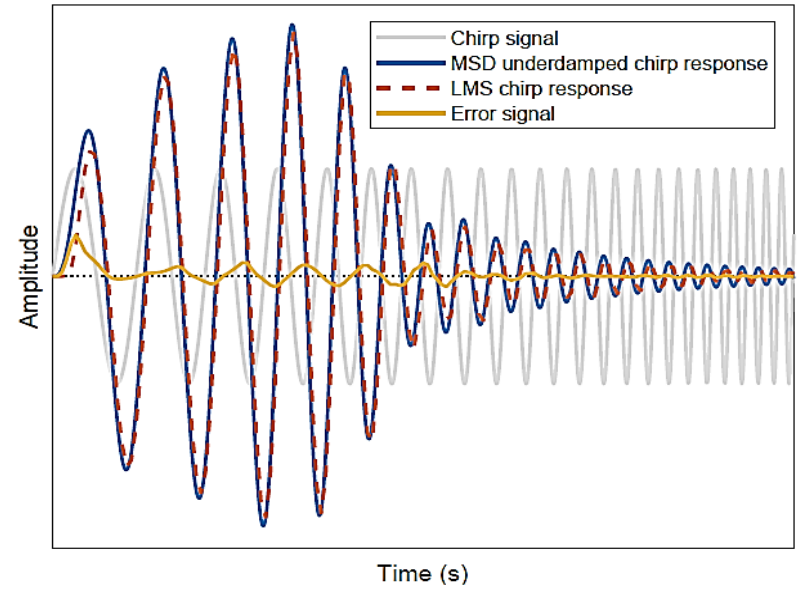

Fig. 9 Response of plant and adaptive filter for chirp excitation

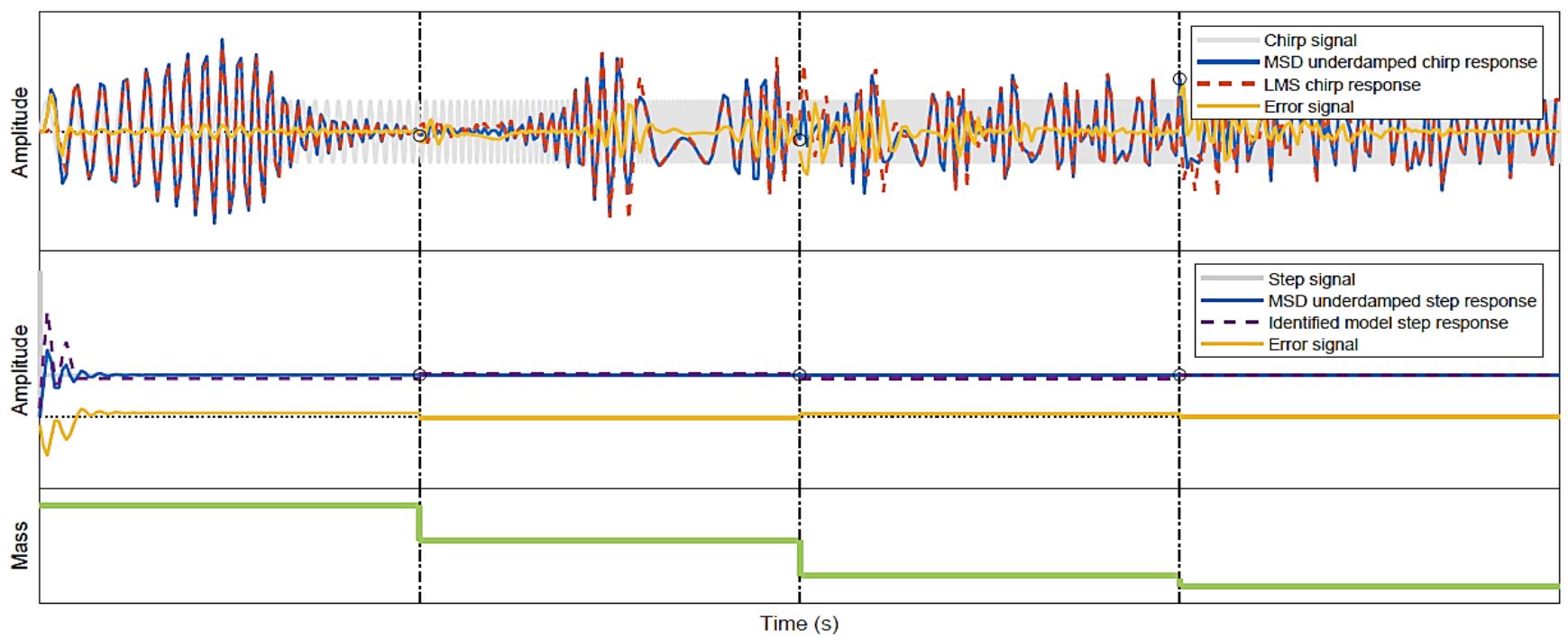

Fig. 10 Chirp response of varying dynamic system and validation of model using step response

such that both outputs matches. From the weights, for which error converges to zero, the model is identified. Two cases of identification has been performed in this work. Case (i) -
Fig. 9. From the results obtained it can be inferred that, the identified model using LMS algorithm based adaptive filter 
identification represents the actual dynamics of the system with an excellent fit.

Case (ii): In this case, the mass of the system has been changed at regular intervals of time as shown in Table 1. Response of the system for chirp excitation and validation using step response are shown in Fig. 10.

\section{CONCLUSION AND Future SCOPE}

From this study it can be understood that a system with varying dynamics can be identified using LMS based adaptive filter technique. Identification of MSD system, with two cases have been successfully performed. From the simulation results it can be observed that the model obtained from adaptive filter represents the dynamics of original system with a good fit. The same technique of identification

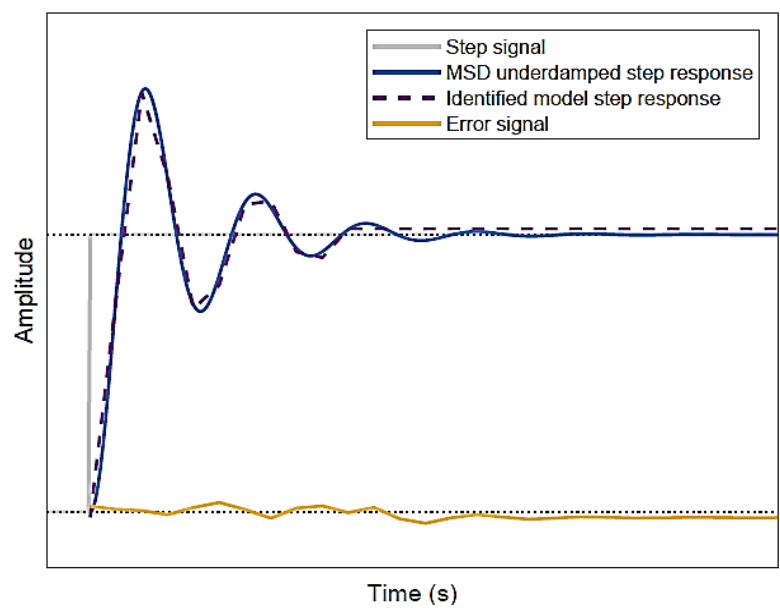

Fig 9. Model validation

can be later used for real time systems with varying dynamics.

\section{Acknowledgment}

The authors would like to acknowledge the support provided by Manipal Institute of Technology, MAHE, for performing this work.

\section{REFERENCES}

[1] P. Kshirsagar, D. Jiang and Z. Zhang, "Implementation and Evaluation of Online System Identification of Electromechanical Systems Using Adaptive Filters," in IEEE Transactions on Industry Applications, vol. 52, no. 3, pp. 2306-2314, May-June 2016.

[2] N. Hallemans, R. Pintelon, D. Peumans, J. Lataire, "Improved frequency response function estimation by Gaussian process regression with prior knowledge," IFAC-PapersOnLine, Volume 54, Issue 7, 2021, Pages 559-564.

[3] M. Algreer, M. Armstrong and D. Giaouris, "Active Online System Identification of Switch Mode DC-DC
Power Converter Based on Efficient Recursive DCDIIR Adaptive Filter," in IEEE Transactions on Power Electronics, vol. 27, no. 11, pp. 4425-4435, Nov. 2012.

[4] A. A. M. Khalaf, M. M. Ibrahim and H. F. A. Hamed, "Performance study of adaptive filtering and noise cancellation of artifacts in ECG signals," 2015 17th International Conference on Advanced Communication Technology (ICACT), Seoul, 2015, pp. 394-401.

[5] K. R. Godfrey et.al., "Comparison of perturbation signals for linear system identification in the frequency domain," in IEE Proceedings - Control Theory and Applications, vol. 146, no. 6, pp. 535-548, Nov 1999.

[6] Peter young and Ronald J. Patton. "Comparison of test signals for aircraft frequency domain identification", in Journal of Guidance, Control, and Dynamics, vol. 13, No. 3, pp. 430-438, 1990.

[7] S.C. Douglas and M. Rupp, “Convergence Issues in the LMS Adaptive Filter," in The DSP Handbook, V.J. Madisetti and D. Williams, eds. (Boca Raton, FL: CRC/IEEE Press, 1998), Chapter 19.

[8] F. Demourant and G. Ferreres, "A frequency domain identification-control approach for a flexible aircraft," Proceedings of the International Conference on Control Applications, 2002, pp. 126-131 vol.1

[9] A. Novak, L. Simon, F. Kadlec and P. Lotton, "Nonlinear System Identification Using Exponential Swept-Sine Signal," in IEEE Transactions on Instrumentation and Measurement, vol. 59, no. 8, pp. 2220-2229, Aug. 2010.

[10] S. E. Saarakkala and M. Hinkkanen, "Identification of Two-Mass Mechanical Systems Using Torque Excitation: Design and Experimental Evaluation," in IEEE Transactions on Industry Applications, vol. 51, no. 5, pp. 4180-4189, Sept.-Oct. 2015.

[11] N. Kong et.al., "Automated System Identification of Digitally-Controlled Multi-phase DC-DC Converters," 2009 Twenty-Fourth Annual IEEE Applied Power Electronics Conference and Exposition, Washington, DC, 2009, pp. 259-263.

[12] D. Martin et.al., "Wide bandwidth system identification of AC system impedances by applying pertubations to an existing converter," 2011 IEEE Energy Conversion Congress and Exposition, Phoenix, AZ, 2011, pp. 25492556.

[13] V. R. Puttige and S. G. Anavatti, "Real-Time Neural Network Based Online Identification Technique for a UAV Platform," in CIMCA-IAWTIC'06, Sydney, NSW, 2006, pp. 92-92.

[14] L. Peretti, M. Zigliotto, "Identification of mechanical load for electrical drives commissioning - labeling machine case study", Proc. IEEE EUROCON, pp. 797803, May 2009.

\section{Creative Commons Attribution License 4.0 (Attribution 4.0 International, CC BY 4.0)}

This article is published under the terms of the Creative Commons Attribution License 4.0 https://creativecommons.org/licenses/by/4.0/deed.en_US 